\title{
Influence of 24-Hour Sleep Deprivation on Respiration in Lambs
}

\author{
PATRICK LÉTOURNEAU, THÉOPHILE NIYONSENGA, ÉMILIE CARRIER, \\ ESTELLE PRAUD, AND JEAN-PAUL PRAUD \\ Respiratory Research Unit, Departments of Pediatrics and Physiology [P.L., E.C., E.P., J-P.P.], \\ Department of Public Health [T.N.], University of Sherbrooke, Sherbrooke, Quebec, Canada
}

\begin{abstract}
The aim of this study was first to examine the effects of 24-h sleep deprivation on apnea index and duration in lambs. The effects on sleep architecture and sigh and swallowing indices were also studied. The impact of postnatal maturation on all measured variables was assessed by studying two different age groups. Twelve lambs (six aged 1-2 d and six aged 23-24 d on the day of surgery) were chronically instrumented for polysomnographic recordings including sleep state assessment, nasal flow, diaphragm electromyogram, and glottal constrictor muscle electromyogram. Two recordings, one control and one after 24-h sleep deprivation, were performed in all lambs. Results show that the effects of sleep deprivation predominate in rapid eye movement sleep in the younger group, with increased rapid eye movement sleep proportion and apnea, sigh, and swallowing index. Our results in lambs suggest that the consequences of
\end{abstract}

\section{ABSTRACT}

sleep deprivation upon respiration are predominant early after birth. Although the potential relationship of these observations to neonatal apneas and sudden infant death syndrome has yet to be defined, awareness of the effects of sleep deprivation is important for neonatal care. (Pediatr Res 52: 697-705, 2002)
TA, thyroarytenoid muscle
EMG, electromyogram
Di, diaphragm
ECoG, electrocorticogram
EOG, electrooculography
REM, rapid eye movement
nREM, non-rapid eye movement

Abbreviations
Numerous studies have shown the physiologic importance and the clinical relevance of exploring the intimate link between sleep and control of breathing. Several studies in adults have shown that sleep deprivation has an impact on both sleep architecture and control of breathing, with the potential to increase breathing disturbances. In adult dogs, the arousal response to hypoxia, hypercapnia, or laryngeal stimulation is decreased after sleep fragmentation (1). In awake adult humans, sleep deprivation leads to a decrease in the ventilatory response to hypoxia (2), in the amplitude of genioglossus EMG (3), and in inspiratory muscle endurance (4). On the other hand, a recent study (5) was unable to confirm the decrease in ventilatory response to hypercapnia (6).

There is growing awareness that postnatal maturation of cardiorespiratory control is intimately linked to ontogeny of sleep-wake cycles (7). Circumstances leading to sleep depri-

Received July 4, 2001; accepted April 19, 2002.

Correspondence: Jean-Paul Praud, M.D., Ph.D., Departments of Pediatrics and Physiology, Université de Sherbrooke, Sherbrooke J1H5N4, Quebec, Canada; e-mail: Jp.Praud@usherbrooke.ca

Supported by the Canadian Institutes of Health Research, grant MT 15558. P.L. was a scholar of the "Fonds pour la Formation de Chercheurs et l'Aide à la Recherche". J.-P.P. is a senior clinician-scientist of the "Fonds de la Recherche en Santé du Québec."

DOI: 10.1203/01.PDR.0000032069.68192.5D vation in neonates, such as stays in neonatal intensive care units, are not rare. Studies on the effects of sleep deprivation in the early postnatal period are therefore especially relevant for understanding neonatal cardiorespiratory control disorders, including apneas and certain cases of sudden infant death syndrome (7). To our knowledge, only two studies have been specifically designed to examine the influence of a short sleep deprivation period on breathing in human infants aged a few months $(8,9)$. Observations from these studies suggested an increase in apnea index and upper airway obstruction.

The present experiments were designed to examine the effects of prolonged (24-h) sleep deprivation in nonsedated, freely moving lambs, using our unique, recently described telemetry polysomnographic equipment. We first aimed at confirming in the lamb previous observations in human neonates where sleep deprivation modified sleep architecture and increased apneas $(8,9)$. Secondly, motivated by recent observations by us and others that sighs $(10,11)$ and nonnutritive swallows (12) can greatly interfere with breathing and oxygenation in the neonatal period, we tested the hypothesis that sighs and swallowing activity are in fact altered after sleep deprivation. Thirdly, due to the importance of glottal constrictor muscle activity to defend functional residual capacity (13) and 
lung volume during apnea (14) in neonates, we studied the effect of sleep deprivation upon TA muscle EMG (TA is a glottal constrictor). Finally, given the major importance of postnatal maturation on cardiorespiratory control and ontogeny of sleep-wake cycles (7), we tested the hypothesis that the effects of sleep deprivation vary according to postnatal age (a few days versus a few weeks of age). Results show that the effects of 24-h sleep deprivation on breathing are predominant in the younger lambs.

\section{METHODS}

\section{Animals}

Twelve mixed-breed term lambs were studied. Lambs were separated in two groups, according to their postnatal age. The first group was composed of six young lambs aged $1.2 \pm 0.7 \mathrm{~d}$ (mean $\pm \mathrm{SD}$ ) with a mean weight of $4.6 \pm 0.3 \mathrm{~kg}$ on the day of surgery. The second group consisted of six older lambs, aged $23.3 \pm 1.4 \mathrm{~d}$, with a mean weight of $8.1 \pm 1.2 \mathrm{~kg}$ on the day of surgery. Age groups were chosen in relation to postnatal carotid body maturation, young lambs being studied before and older lambs after maturation of carotid body function (15). The study was conducted in nonsedated lambs to avoid undesirable cardiorespiratory effects induced by sedatives and anesthetics. The protocol of the study was approved by the ethics committee of the University of Sherbrooke for animal care and experimentation.

\section{Surgical Preparation}

Surgery was performed under general anesthesia (enflurane $2-3 \%+\mathrm{N}_{2} \mathrm{O} 30 \%$ ), after premedication by atropine sulfate $(100 \mu \mathrm{g} / \mathrm{kg}$ s.c.), and intramuscular ketamine $10 \mathrm{mg} / \mathrm{kg}$ (Ketaset, Wyeth-Ayerst Canada, St-Laurent, QC, Canada) and midazolam $100 \mu \mathrm{g} / \mathrm{kg}$ (Versed, Hoffman-La Roche, Mississauga, ON, Canada). Bipolar enameled chrome wire electrodes (0.1 $\mathrm{mm}$ diameter, Chromel, GTSM, Castelnaudary, France) were inserted into the TA and Di muscles for EMG recording, as previously reported (16). Briefly, an EMG electrode was sewn under direct vision into both TA muscles through a small window made on each side of the thyroid cartilage. For the Di, two electrodes were sewn into the costal portion of the right $\mathrm{Di}$ after a small thoracotomy. Correct positioning of electrodes in laryngeal and diaphragmatic muscles was always verified at autopsy. One hole was drilled in each parietal bone for ECoG recording, $1 \mathrm{~cm}$ from the midline. Two electrodes constructed from enameled chrome wire threaded through a nylon screw and coiled at its bare end were screwed in the holes in such a way that the active part of the electrode was apposed on the dura matter. EOG were recorded with two cup electrodes (Neotrode, ConMed Andover Medical, Haverhill, MA, U.S.A.) glued subcutaneously at the outer and inner canthi, respectively, of the right eye. ECG signals were recorded from two cup electrodes placed $2 \mathrm{~cm}$ apart on the anterior thoracic wall. Finally, one cup electrode glued more anteriorly on the skull served as a ground. All leads were subcutaneously tunneled to exit on the back. Nasal airflow was recorded using a thermocouple wire (iron/constantan, type J; Omega Engineering,
Stamford, CT, U.S.A.) glued to the side of one nostril; accuracy of this method for apnea detection in lambs has been verified previously by simultaneous recording of diaphragm EMG (14).

Lambs returned to their mothers after arousal from anesthesia. Postoperative care systematically included analgesia with one intramuscular injection of buprenorphine $0.005 \mathrm{mg} / \mathrm{kg}$ (Buprenex, Reckitt \& Coleman Pharmaceuticals, Richmond, VA, U.S.A.), and antibiotic therapy with penicillin (Duplocillin LA, Intervet Canada, Whitby, ON, Canada) and gentamicin (Gentocin, Schering Canada, Pointe-Claire, QC, Canada).

\section{Measurement Apparatus}

To obtain data from prolonged recordings (with periods of wakefulness and sleep) under more natural conditions, we recently designed a radiotelemetry device that enables us to study free moving lambs $(17)$. The transmitter $(3.5 \times 2.5 \times 0.8$ $\mathrm{cm})$ used in the study included eight differential channels designed for EMG (four channels), nasal flow, ECG, EOG, and $\mathrm{ECoG}$, each channel having its own differential preamplifier and high-pass filter. The eight signals were transmitted on the same radio frequency using temporal multiplexing. The resulting signal was demultiplexed by the receiver to rebuild the eight original signals. For more flexibility, each channel possessed its own variable gain amplifier and polarity selector at the receiver level. Finally, the eight signals were sent from the receiver to the acquisition system. The raw EMG signals were moving time averaged by the acquisition software (AcqKnowledge version 3.2, Biopac Systems, Santa Barbara, CA, U.S.A.). Raw and integrated EMG, EOG, ECoG, ECG, and nasal flow signals were recorded on a Power Macintosh 7300 using a computerized acquisition device (MP100A, Biopac Systems).

\section{Design of the Study}

All 12 lambs (six young and six older lambs) underwent both control and post 24-h sleep deprivation polysomnographic recording. Surgery was performed at 1 to $2 \mathrm{~d}$ of age for the younger group and at 23 to $26 \mathrm{~d}$ of age for the older group. The first recording was performed $48 \mathrm{~h}$ after surgery. To prevent the potential confounding effects of both the early postoperative period and the postnatal maturation between the first and second recordings (especially in the younger group), block randomization was used to ensure that three lambs in each group had a control recording before and the remaining three lambs after $24 \mathrm{~h}$ postdeprivation. Thus, in both groups, if we consider that surgery was performed at $\mathrm{d} 1$ in all six lambs, three lambs had a control recording at $d 3$, sleep deprivation at $\mathrm{d} 4$ and a postdeprivation recording at $\mathrm{d} 5$. The remaining three lambs had sleep deprivation at $\mathrm{d} 3$, a postdeprivation recording at $\mathrm{d} 4$, and a control recording at d 5. Adequacy of such a design was verified after completion of the study by confirming that results of the control studies were not statistically different if obtained before or after the sleep deprivation recording. In all lambs, recordings were performed between 1700 and $2300 \mathrm{~h}$. Sleep deprivation was induced between $1700 \mathrm{~h}$ and $1700 \mathrm{~h}$ the next day by an investigator who was present throughout the 24-h sleep deprivation period. The lamb stood 
in a Plexiglas box close to the observer or walked about on the floor of the laboratory throughout the deprivation period. Gentle manual or auditory stimulation was used as required to prevent the lamb from lying down and/or closing its eyes.

Just before each recording, the radiotelemetry transmitter was connected to the electrode leads and attached to the lamb's back. Lambs were studied in conditions as close to normal as possible, i.e. with the ewe and other lambs in the pen where they all lived since their arrival in our animal quarters. Use of our telemetry equipment allowed us to study nonsedated, freely moving lambs able to feed and sleep ad libitum. Signals from the transmitter were transmitted to an antenna placed in the pen and then forwarded to our laboratory for remote acquisition and subsequent data analysis. Lambs were continuously monitored using infrared video cameras placed in the recording room, an observer being always present in the laboratory to note all events occurring during recordings. Usual light cycle, i.e. light extinction between 1800 and $0600 \mathrm{~h}$, was kept throughout the experimental days, together with constant room temperature $\left(22^{\circ} \mathrm{C}\right)$ and humidity $(70 \%)$.

\section{Data Analysis}

Variables: definitions and measurements. All signals were carefully observed and analyzed throughout the recordings according to states of arousal of the lamb. Variables recorded for each lamb are described below. States of arousal were identified by usual electrophysiological and behavioral criteria: 1) wakefulness, from the presence of high-frequency, lowvoltage ECoG pattern with open eyes and occasional body movements; 2) REM sleep, from the presence of highfrequency, low-voltage ECoG pattern, with bursts of REM and body twitches; and 3) nREM sleep, from slow-frequency, high-voltage ECoG pattern with closed eyes, absence of REM and body movements. Arousal from nREM sleep was characterized by sudden disappearance of high amplitude waves in the EcoG for at least $3 \mathrm{~s}$. Arousal from REM sleep was recognized by direct observation of the lamb and disappearance of intense EOG activity. Apneas were defined as the absence of nasal airflow for at least $3 \mathrm{~s}$ and were classified as central, mixed, or obstructive from diaphragm EMG (respectively absent or present during part or throughout apnea). The apnea index (number of apneas per hour) and duration of each apnea were calculated for the recording period as a whole and for each state of arousal. We also noted whether apneas were preceded by an event such as a swallow or a sigh, or if they occurred suddenly. Heart rate and breathing frequency were measured at 30-s intervals, 1 min after onset of each epoch for wakefulness and nREM sleep, and $30 \mathrm{~s}$ after onset of each REM sleep epoch. Furthermore, heart rate was measured during and after apneas, bradycardia being defined as a fall in heart rate of at least 30\% (14). Sigh and swallowing indices (number of sighs or swallows per hour) were calculated for the recording period as a whole and for each state of arousal. Sighs were defined as an inspiratory diaphragm EMG activity with an amplitude at least 2-fold that of the preceding three breaths. Swallows were easily recognized as an abrupt phasic TA EMG activity of short duration and large amplitude. TA EMG was analyzed throughout all recordings. Firstly, aside from apneas, calculations of percentage of breathing cycles with expiratory TA were made at 30-s intervals for each state of arousal as described above. Secondly, we noted whether TA EMG was present during apneas, and whether it was continuous throughout apneas.

Research queries. The first outcomes of our study were the apnea index and apnea duration. Secondary outcomes were duration of states of arousal, respiratory rate and heart rate, sigh and swallowing indices, heart rate with apneas, and TA EMG during ventilation as well as during apneas. Two potential predictors of all variables to be controlled were the state of arousal and maturation (postnatal age) of the lamb. Specific questions were as follows: Does sleep deprivation 1) increase apnea index and/or apnea duration; 2) increase percentage of total sleep time and REM sleep; 3 ) increase mean duration of REM epochs; 4) increase bradycardia-related apnea; 5) modify breathing frequency or heart rate; 6) modify sigh and swallowing indices; 7) decrease the number of breathing cycles with TA expiratory EMG; or 8) decrease the number of apneas accompanied by continuous TA EMG?

Statistical analysis. Statistical analyses were performed under the close guidance of a biostatistician (T.N.). Two different types of statistical analyses were performed: descriptive and inferential. Firstly, all data were summarized in terms of mean and proportion by age group (young or older) and by state of arousal (wakefulness, nREM, and REM). Results were expressed as mean $\pm \mathrm{SD}$. Secondly, different specific questions from the above research queries were asked. When the correlation structure did not have to be taken into account, $t$ test or Wilcoxon signed ranked test for independent or paired samples were used, normality having been tested beforehand by the nonparametric Kolmogorov-Smirnov test. Complexity of the data structure, with two age groups, three states of arousal, and two measurement times (before and after sleep deprivation), did require at times the use of a generalized regression model applied to correlated data (18). All analyses were performed using either SAS release 6.12 (SAS Institute, Cary, NC, U.S.A.) or SPSS version 8 (SPSS Science, Chicago, IL, U.S.A.). A $p$ value $<0.05$ was considered significant.

\section{RESULTS}

All twelve lambs were studied before and after sleep deprivation, in a randomized order. Mean duration of recordings was $4.46 \pm 0.15 \mathrm{~h}$.

Sleep architecture. Results on sleep architecture are shown in Figure $1 A$. In control conditions (without sleep deprivation), younger lambs spent more time asleep than older lambs $(30 \pm$ $11 \%$ versus $14 \pm 3 \%$, respectively, $p=0.015$ ). Moreover, whereas older lambs spent an identical percentage of time in nREM and REM sleep, younger lambs spent more time in nREM than in REM sleep ( $61 \%$ and $39 \%$ of total sleep time, respectively, $p=0.048$ ). Sleep deprivation was responsible for a significant increase in the percentage of time spent asleep in both younger (from $30 \pm 11 \%$ to $56 \pm 12 \%, p=0.002$ ) and older (from $14 \pm 3 \%$ to $35 \pm 5 \%, p=0.03$ ) lambs. However, younger lambs still spent significantly more time asleep than 
older lambs $(p=0.009)$. Both nREM and REM sleep significantly increased in younger and older lambs after sleep deprivation, the increase being more important for REM sleep in younger lambs. Finally, the percentage of sleep time spent in REM sleep after sleep deprivation was not significantly different in the younger $(56 \%)$ and the older lambs $(46 \%)$.

Results on mean duration of wakefulness and nREM and REM sleep episodes are shown in Figure $1 B$. In control conditions, the only statistically significant difference was a longer mean duration of wakefulness episodes in older lambs (36 $\pm 8 \mathrm{~min})$ compared with younger lambs $(13 \pm 6 \mathrm{~min}, p<$ 0.0001 ). Sleep deprivation was responsible for a significant decrease in mean duration of wakefulness episodes in both younger and older lambs $(p=0.001)$. Consequently, duration of wakefulness episodes was no longer significantly different in younger and older lambs $(5.7 \pm 3.0 \mathrm{~min}$ and $11.2 \pm 5.6 \mathrm{~min}$, respectively, $p=0.065$ ). Moreover, mean duration of REM sleep episodes significantly increased in younger lambs $(p=$ 0.027).

Characteristics of apneas. A total of 456 central apneas (see example in Fig. 2) were recorded in 12 lambs. No mixed or obstructive apneas were observed in any of the lambs. In control conditions, all states of arousal taken together, younger lambs tended to have a slightly higher mean apnea index than older lambs $(3.6 \pm 3.2 / \mathrm{h}$ and $3.0 \pm 2.3 / \mathrm{h}$, respectively, NS) (Fig. 3). Moreover, whereas apnea index tended to be higher in nREM compared with REM sleep in younger lambs (NS), the reverse was observed in older lambs (NS). After sleep deprivation, all states of arousal taken together, younger lambs had a higher apnea index than older lambs $(5.1 \pm 3.0 / \mathrm{h}$ and $3.2 \pm$ $2.5 / \mathrm{h}$, respectively, $p=0.009$ ). Indeed, only younger lambs significantly increased their apnea index after sleep deprivation $(p=0.001)$ (Fig. 3). More precisely, apnea index tended to increase in wakefulness and nREM sleep in younger lambs, and increased more than 2-fold in REM sleep $(1.7 \pm 2.2 / \mathrm{h}$ to $4.3 \pm 2.4 / \mathrm{h}, p=0.018$ ). No significant differences were observed for apnea duration, with a mean range between 5 and $8 \mathrm{~s}$ in both groups of lambs, in control conditions and after sleep deprivation. Finally, no bradycardia was observed during or after apneas either before or after sleep deprivation in both younger and older lambs.

In control conditions, regardless of the state of arousal, most apneas were preceded by a sigh in both younger and older lambs (111/113 and 83/103 apneas, respectively). Sleep deprivation did not induce any modification in this proportion (130/145 and 78/95 apneas, respectively). Interestingly, however, the total number of apneas not preceded by a sigh recorded in REM in younger lambs increased from 0 to 10 after sleep deprivation. Finally, of all the observed apneas, only one was preceded by a swallow.

Breathing frequency. In control conditions, breathing frequency in younger lambs was about 2-fold that of older lambs in all states of arousal $(p<0.0001)$. Sleep deprivation had no significant influence on breathing frequency regardless of postnatal age or state of arousal. Moreover, breathing frequency in younger lambs was still about 2 -fold over that of older lambs, regardless of the state of arousal $(p<0.0001)$ (Fig. $4 A$ ).

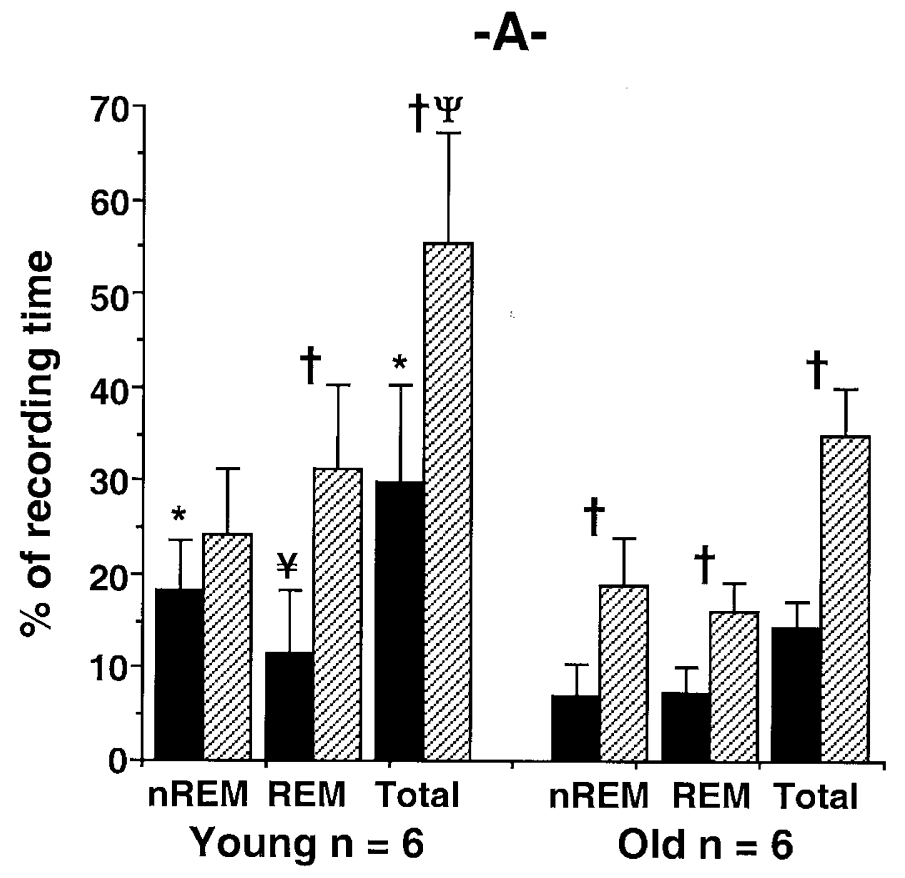

\section{-B-}

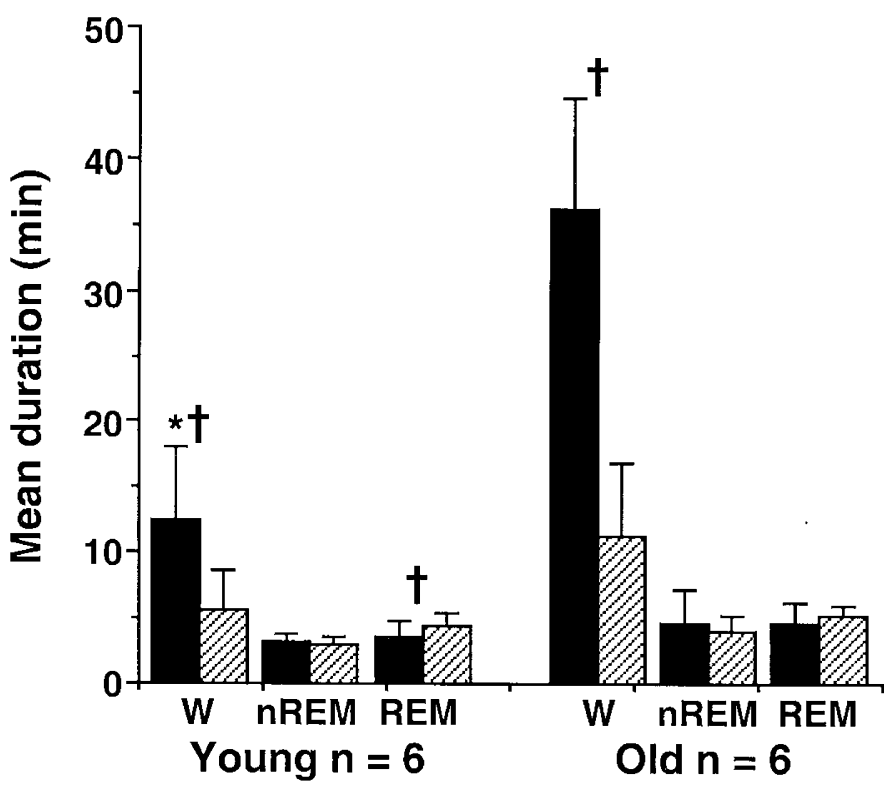

Figure 1. Sleep/wakefulness architecture before and after sleep deprivation for both age groups. $(A)$ percentage of time spent in different states of arousal and $(B)$ mean duration of each episode. ${ }^{*} p<0.015$, young $v s$ older lambs (baseline); $\Psi p=0.009$, younger $v s$ older lambs (postsleep deprivation); $¥ p<$ 0.05, REM vs nREM sleep (control); †p $<0.032$, control vs postsleep deprivation. Filled bars, control; shaded bars, postsleep deprivation.

Heart rate. In control conditions, all states of arousal taken together, heart rate was higher in younger lambs (177 \pm $\left.20 \cdot \mathrm{min}^{-1}\right)$ than in older lambs $\left(133 \pm 19 \cdot \mathrm{min}^{-1}\right)(p<0.0001)$ (Fig. $4 B$ ). Sleep deprivation significantly increased heart rate in younger lambs when all states of arousal were taken together $(p$ $<0.0001)$ and in older lambs during wakefulness $(p=0.0002)$ 
TA

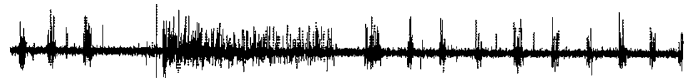

$\int T A$

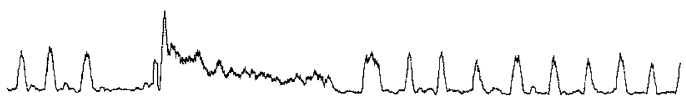

Di

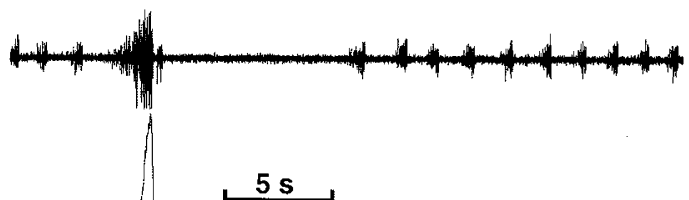

$\int \mathrm{Di}$

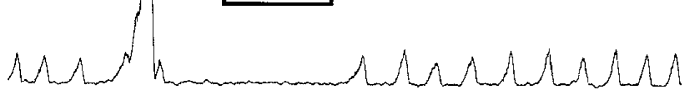

$\mathbf{F}$

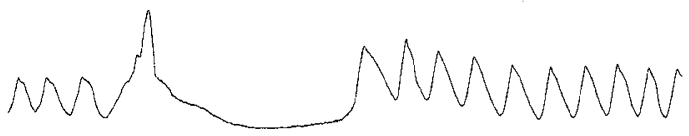

ECG

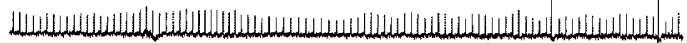

\section{ECoG}

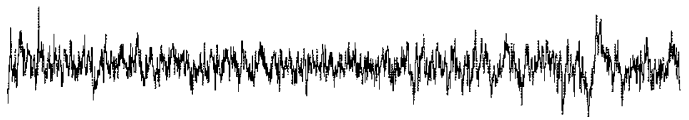

\section{EOG}

Figure 2. Control polysomnographic recording in a 3-d-old lamb showing central apnea after a sigh during nREM sleep. TA, raw TA muscle EMG (glottal adductor); $\int T A$, moving time averaged TA EMG; $D i$ and $\int D i$, raw and integrated diaphragm EMG; $F$, nasal airflow. Note the presence of TA EMG throughout postsigh apnea.

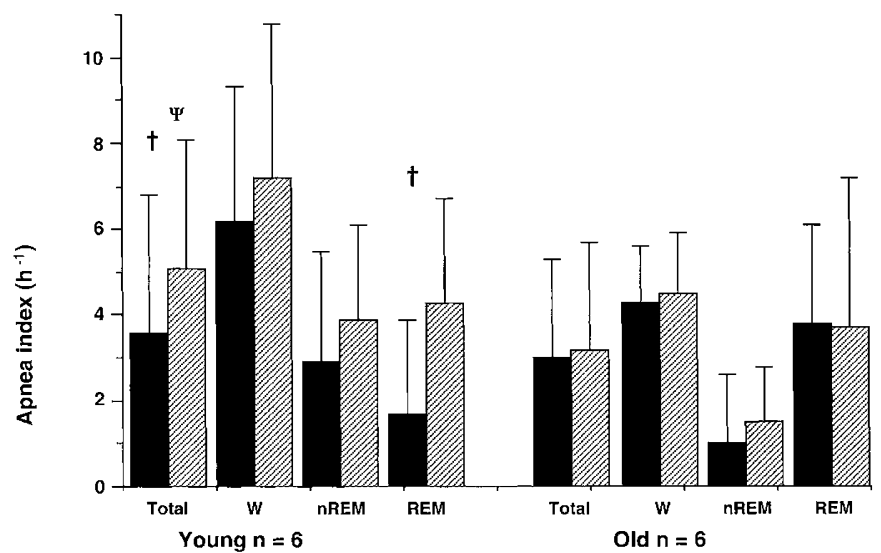

Figure 3. Apnea index before and after sleep deprivation for both age groups and for each state of arousal. $\Psi p=0.009$, younger $v s$ older lambs (postsleep deprivation); $\uparrow p<0.018$, control $v$ s postsleep deprivation. Filled bars, control; shaded bars, postsleep deprivation.

and nREM sleep ( $p=0.003$ ) (Fig. $4 B)$. Moreover, heart rate remained higher in younger lambs after sleep deprivation $(p<$ 0.0001) (Fig. 4B).

Sigh index. In control conditions, all states of arousal taken together, sigh index tended to be higher in younger lambs (7.1
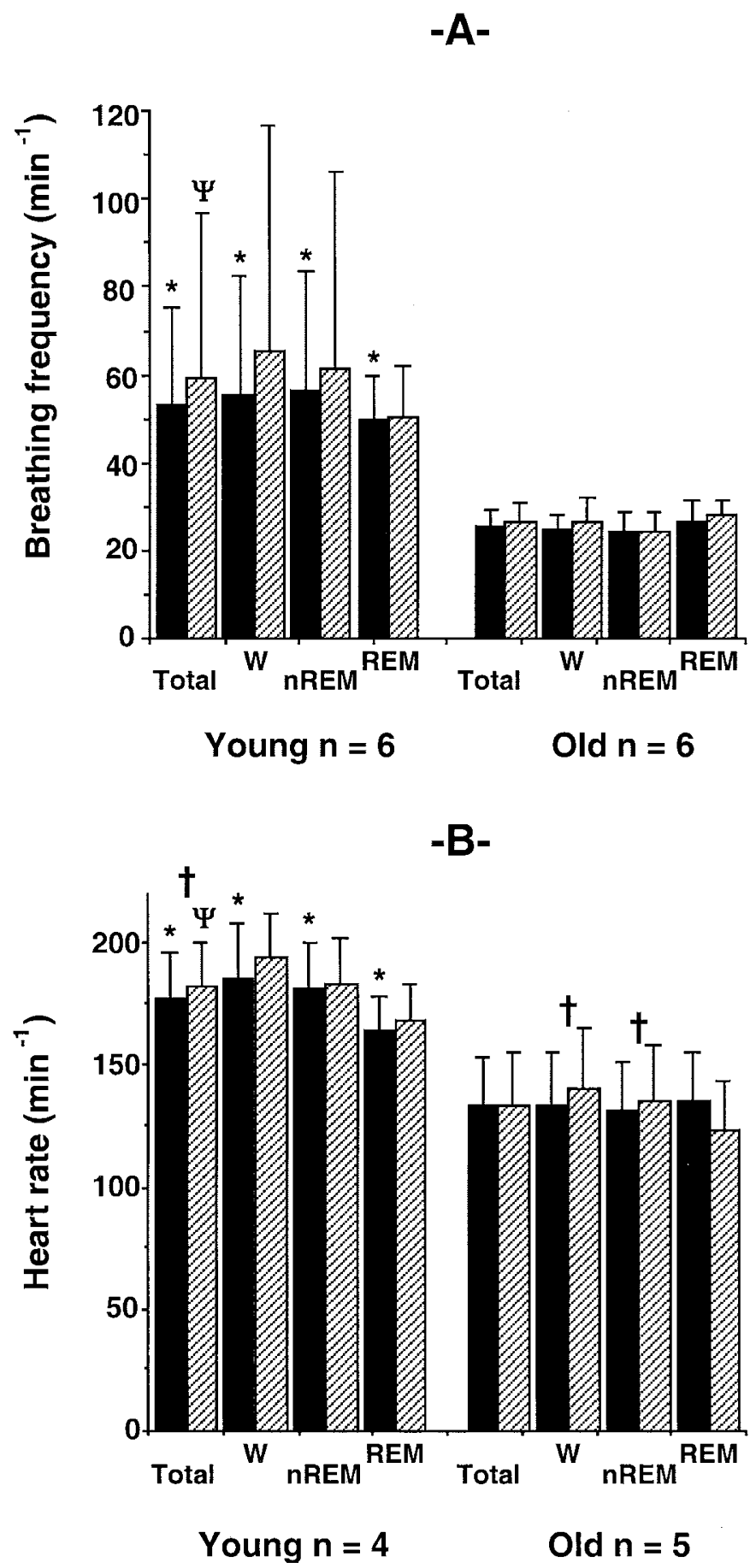

Figure 4. (A) Respiratory rate and $(B)$ heart rate before and after sleep deprivation for both age groups and for each state of arousal. $* p<0.0001$, younger $v s$ older lambs (control); $\Psi p<0.0001$, younger $v s$ older lambs (postsleep deprivation); $\dagger p<0.004$, control vs postsleep deprivation. Filled bars, control; shaded bars, postsleep deprivation.

$\left.\pm 5.3 \cdot \mathrm{h}^{-1}\right)$ than in older lambs $\left(3.7 \pm 3.3 \cdot \mathrm{h}^{-1}\right)$, with a significant difference in wakefulness only $(p=0.015)$ (Fig. $5 A$ ). Moreover, in both groups of lambs, the highest sigh index was observed during wakefulness and the lowest in nREM sleep. After sleep deprivation, all states of arousal taken together, the sigh index was even higher in younger lambs than in older lambs $\left(10.6 \pm 6.4\right.$ versus $\left.4.2 \pm 3.3 \cdot \mathrm{h}^{-1}, p<0.0001\right)$. 
More precisely, sigh index increased in REM sleep in younger lambs $(p<0.001)$, but decreased in older lambs $(p=0.042)$.

Swallowing index. In control conditions, all states of arousal taken together, swallowing index was lower in younger than in older lambs $\left(50.2 \pm 30.6 \cdot \mathrm{h}^{-1}\right.$ and $69.4 \pm 34.1 \cdot \mathrm{h}^{-1}$, respectively, $p=0.018$ ). Moreover, swallowing index was the highest in REM sleep and the lowest in nREM sleep in both younger and older lambs (Fig. 5B). A significant difference was observed only between nREM and wakefulness in younger ( $p$ $<0.0001)$ and older lambs $(p=0.002)$. After sleep deprivation, all states of arousal taken together, the difference in swallowing index between younger and older lambs was smaller. Moreover, sleep deprivation tended to increase swallowing index in wakefulness and REM sleep, the increase being significant only in REM sleep in younger lambs $(p=$ $0.010)$ and in wakefulness in older lambs $(p=0.025)$.

TA EMG during ventilation. In control conditions, all states of arousal taken together, phasic expiratory TA EMG tended to be more frequent in older than in younger lambs $(46 \pm 44 \%$ and $27 \pm 36 \%$ of breaths, respectively, $p=0.12$, NS). Phasic expiratory TA EMG was observed during wakefulness and nREM sleep in all lambs, but was virtually absent in REM sleep (Figs. 6 and 7A). Typically, expiratory TA EMG began at the transition between states of arousal; thereafter, it gradually decreased to 0 , or rapidly disappeared when breathing frequency increased. Low-amplitude phasic inspiratory TA EMG was occasionally observed during wakefulness and nREM sleep in both younger and older lambs. Sleep deprivation in both younger and older lambs tended to decrease the number of breaths with TA expiratory EMG in all states of arousal. This was, however, not true in REM sleep in younger lambs where the number of breaths with TA EMG significantly increased (3\% in control conditions versus $9 \%$ after sleep deprivation, $p$ $=0.002)$.

TA EMG during apneas. In control conditions, regardless of the state of arousal, the percentage of apneas with continuous TA EMG (Fig. 2) was more than 2-fold higher in older lambs than in younger lambs (NS) (Fig. 7B). Sleep deprivation appeared to have no influence on the percentage of apneas with TA EMG in younger lambs, but tended to decrease this percentage during wakefulness and nREM sleep (NS) in older lambs, while increasing the percentage of apneas during REM sleep (NS).

\section{DISCUSSION}

The present study allowed us to examine the effects of 24-h sleep deprivation on sleep architecture and various ventilatory parameters in lambs. Several aspects of this study are unique: prolonged sleep deprivation for the neonatal period; use of radiotelemetry equipment allowing performance of recordings in near normal conditions; assessment of maturation effects by recording two age groups; assessment of the effects of sleep deprivation on sigh and swallowing index; and assessment of the effects of sleep deprivation on glottal muscle activity. Results show that the effects of sleep deprivation are predominant in REM sleep during the first few days after birth: increased REM sleep
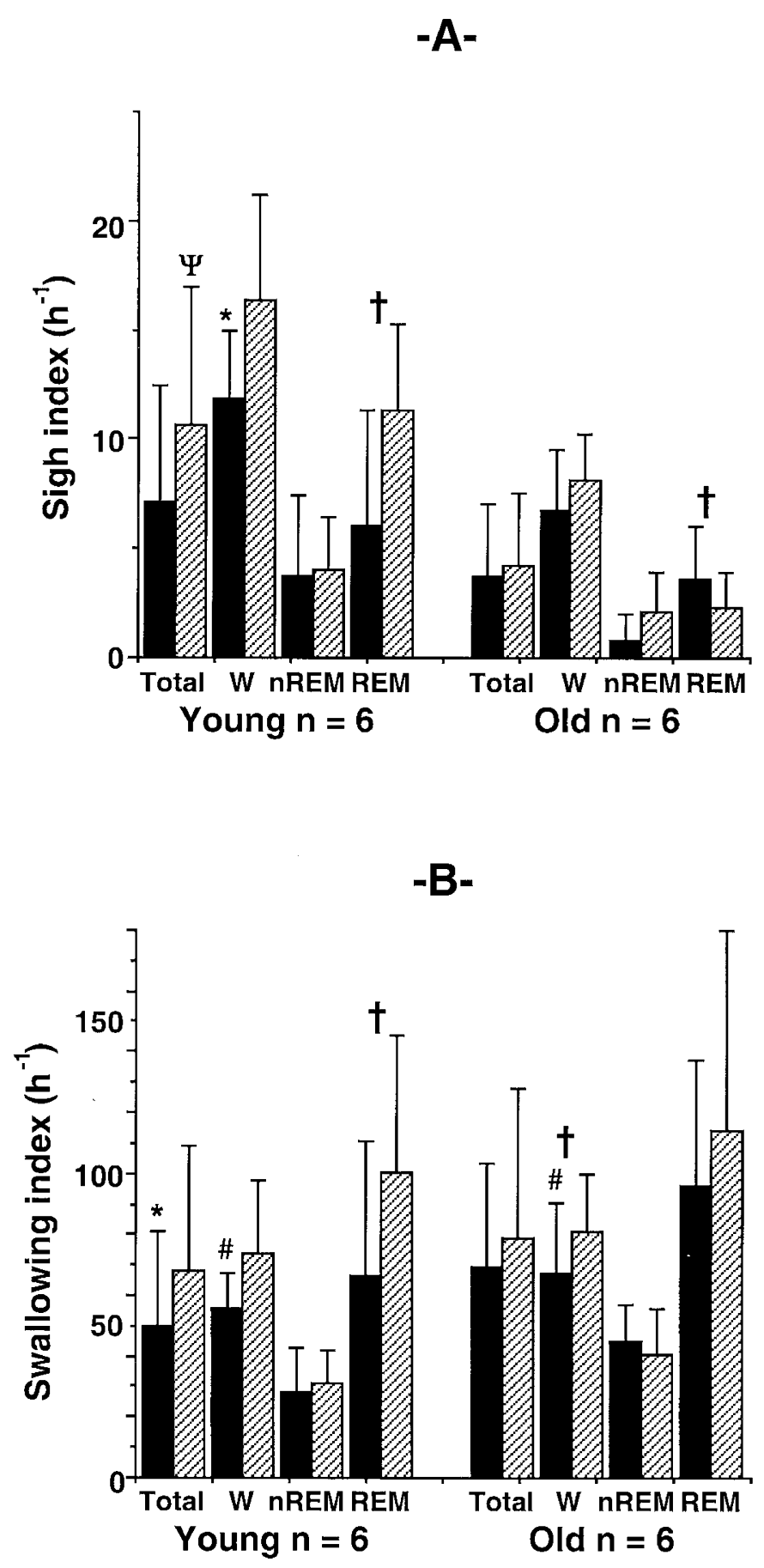

Figure 5. (A) Sigh and (B) swallowing indices before and after sleep deprivation for both age groups and for each state of arousal. ${ }^{*} p<0.018$, younger $v s$ older lambs (control); $\Psi p<0.0001$, younger $v s$ older lambs (postsleep deprivation); $\# p<0.002$, wakefulness $v s$ nREM sleep (control); $\dagger p$ $<0.043$, control vs postsleep deprivation. Filled bars, control; shaded bars, postsleep deprivation.

proportion, doubling of apnea index, and increase in sigh and swallowing indices were specifically observed in younger lambs. The effects of postnatal maturation are shown by the absence of such observations in older lambs. Thus, early postnatal respiratory control is particularly susceptible to sleep deprivation compared with older lambs. 
$-A-$

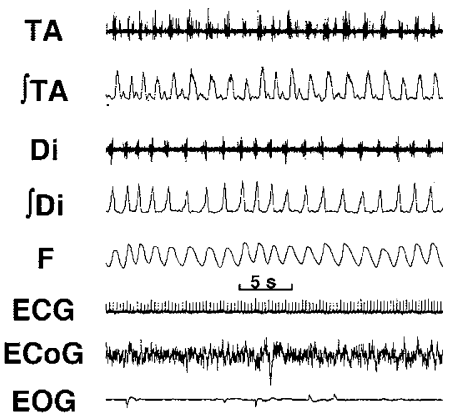

-B-

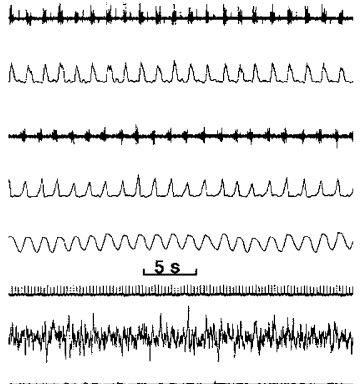

-C-

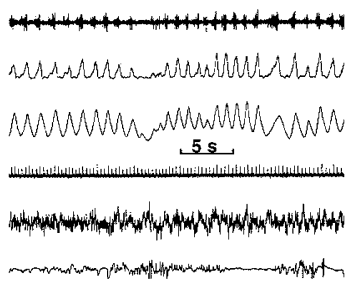

Figure 6. Control polysomnographic recording in a 3-d-old lamb. $(A)$ Wakefulness, $(B)$ nREM sleep, and $(C)$ REM sleep episodes. TA, raw TA muscle EMG (glottal adductor); $\int T A$, moving time averaged TA EMG; $D i$ and $\int D i$, raw and integrated diaphragm EMG; $F$, nasal airflow; Note the absence of TA EMG during REM sleep.

\section{Sleep/Wakefulness Architecture}

The increased percentage of time spent in sleep (nREM + REM sleep) shown in the present study after $24 \mathrm{~h}$ sleep deprivation, regardless of postnatal age, is in agreement with previous data conducted by others in the neonatal period $(8,9$, 19). Of particular note, younger lambs (3-5 d of life) had a proportionally greater increase in REM than in nREM sleep, whereas the opposite was true in older lambs (25-27 d of life). These results are in keeping with a recent study in rat pups showing an increase in nREM sleep proportion, only when sleep deprivation was performed after $20 \mathrm{~d}$ of life (19). Our results obtained in older lambs are also reminiscent of the increased nREM sleep proportion reported after a sleep deprivation of shorter duration in human infants aged $2-6$ mo $(8,9)$. Thus, results obtained to date in different species seem to indicate that, apart from the very first days after birth, sleep deprivation is responsible for an increase in nREM sleep proportion in early mammalian life. The increase in REM sleep proportion observed in younger lambs will have to be confirmed in other species including human infants. Indeed, differences between species such as those described in adult mammals (20) may exist in the early postnatal period.

\section{Influence of Sleep Deprivation on Neonatal Apneas}

In control conditions, apnea index was the highest during wakefulness, in both younger and older lambs. As most apneas were preceded by a sigh, this is at least partly due to the higher sigh index in wakefulness. The increase in apnea index after sleep deprivation observed in younger lambs, especially in REM sleep, is in agreement with results in human infants $(8$, 9). Furthermore, the absence of any increase in apnea index in older lambs, regardless of the state of arousal, is probably related to neurologic maturation. This may especially but not only include maturation of chemical control of ventilation such as the resetting of carotid body function (15), which takes place during the first $2 \mathrm{wk}$ of life in lambs. Changes in lung volumes (21) and maturation of vagal control of ventilation (22), which have been shown to take place within the first $3 \mathrm{wk}$ of postnatal life in lambs, may also partly explain lesser effects of sleep deprivation in older lambs. Thus, we hypothesize that sleep deprivation, before completion of the early postnatal matura- tion of ventilatory control, induces a higher risk of ventilatory instability, especially in REM sleep. This, together with the increase in REM sleep proportion and the increase in REM sleep epoch duration observed in younger lambs, suggests that sleep deprivation may have clinical consequences in the early postnatal period. Of note, however, no epoch of periodic breathing was recorded in any full-term lambs in the present study, even after sleep deprivation. This is in marked contrast with what we have recently observed in preterm lambs (without sleep deprivation) (14).

The absence of any obstructive and mixed apneas in the present study is different from two previous observations made in human infants aged a few months, showing a significant increase in obstructive apneas, particularly during REM sleep (8) or at least a tendency toward an increase in upper airway obstruction in nREM sleep (9). We hypothesize that this is mainly related to species differences, possibly including upper airway anatomy and/or neural control. Indeed, although it is known that mixed/obstructive apneas are frequent in preterm infants (23), we have already reported that only a few mixed and no obstructive apneas were observed in preterm lambs during baseline polysomnographic recordings (14).

\section{Breathing Frequency and Heart Rate}

As previously reported (24), breathing frequency in control conditions was significantly higher in younger than older lambs. Furthermore, we found no influence of sleep deprivation on breathing frequency, irrespective of postnatal age. Whereas sleep deprivation in adult humans has been reported to induce an increase in breathing frequency during REM sleep (25), this was not confirmed in other studies $(5,26)$. Moreover, previous studies in adults did not find any effect of sleep deprivation upon heart rate or plasma catecholamine levels (5, $26,27)$. The slight but significant increase in heart rate found after sleep deprivation in both groups of lambs may represent an increased sympathetic activity specific to the postnatal period.

\section{Sigh and Swallowing Index}

Sigh index. The importance of sighs for neonatal breathing is widely acknowledged. By increasing functional residual 

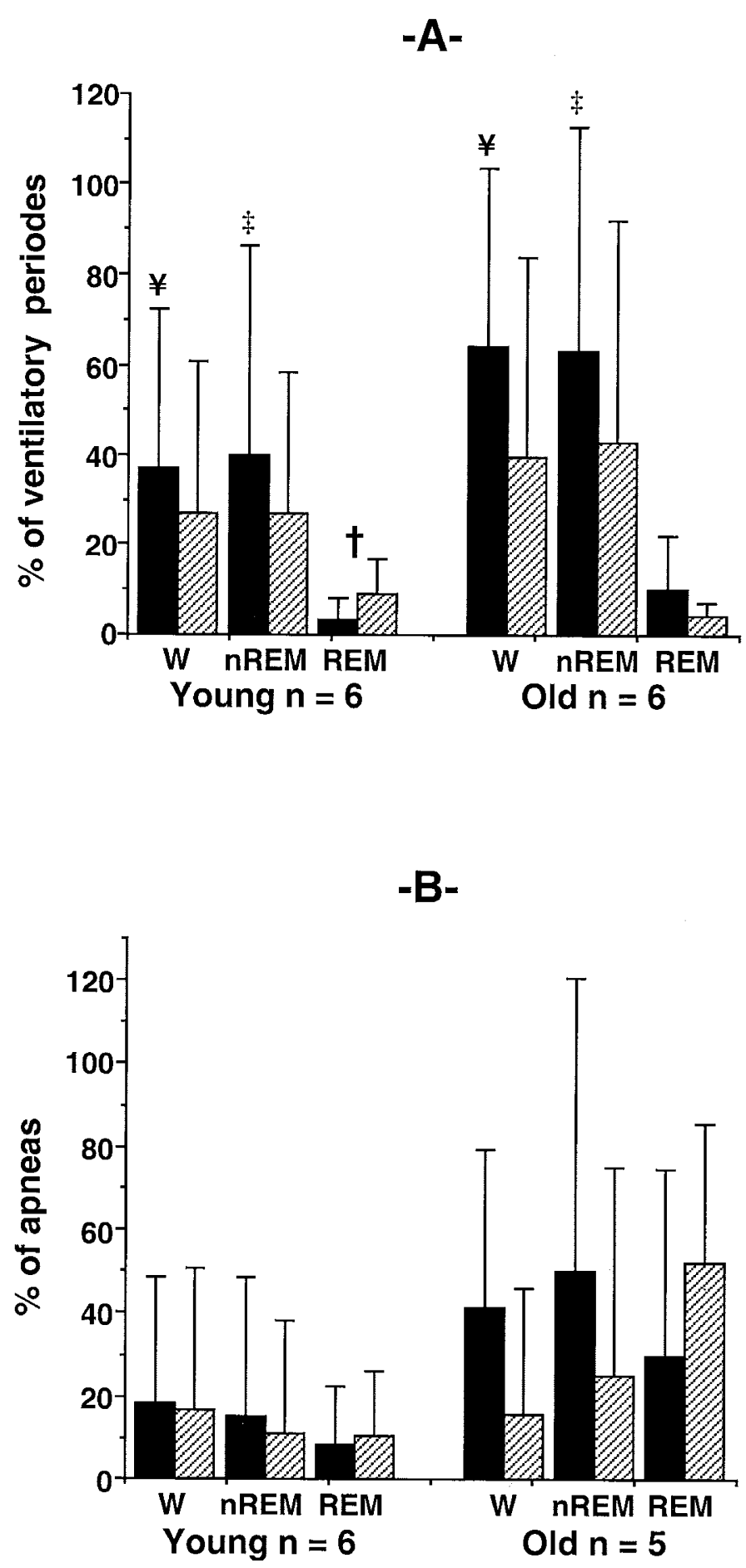

Figure 7. TA muscle activity during ventilatory periods and apneas before and after sleep deprivation for both age groups and for each state of arousal. $(A)$ Percentage of respiratory cycles with expiratory TA EMG activity. $(B)$ Percentage of apneas with continuous TA EMG. $¥ p=0.014$, REM $v s$ nREM sleep (control); $₫ p=0.0003$, wakefulness $v s$ REM sleep (control); $\dagger p<0.002$, control vs postsleep deprivation. Filled bars, control; shaded bars, postsleep deprivation.

capacity and/or redistributing lung volume, sighs will prevent atelectasis and ventilation/perfusion inequality $(10,28)$. This is felt to be especially important in the postnatal period, where defense of functional residual capacity is of concern due to both high rib cage compliance and low lung compliance (11).
In control conditions, our present results in both young and older lambs extend previous results in adult animals and humans (29) showing that sighs occur more often during wakefulness than during sleep. In addition, sighs were observed to be significantly more frequent in younger than in older lambs, similar to previous reports in human infants during sleep (11). Sleep deprivation tended to induce an increase in sigh index, which was significant in REM sleep in younger lambs. To our knowledge, an increase in sigh index after sleep deprivation has not been previously reported. It may represent a compensatory mechanism aimed at increasing functional residual capacity and/or improving lung volume distribution altered by the increased time spent in apnea in REM sleep after sleep deprivation, secondary to the increase in REM sleep duration and apnea index in REM sleep.

Swallowing index. Nonnutritive swallowing is an airwayprotective reflex whose deficiency has been hypothesized to contribute to some cases of sudden infant death syndrome (30). In control conditions, our results in both young and older lambs extend previous results in adults: swallowing activity is decreased with progression from wakefulness to slow wave sleep, but increased in REM sleep (31). The tendency toward an increased swallowing activity, regardless of postnatal age, suggests that sleep deprivation does not inhibit this protective reflex. However, the increase in swallowing index in younger lambs during REM sleep after sleep deprivation is worth noting. We have previously reported that bouts of swallows frequently occur as phasic events of REM sleep in the perinatal period (12), potentially impeding ventilation by inhibiting brainstem respiratory neurons (32).

\section{TA EMG Activity}

Active expiratory laryngeal closure in newborn mammals participates in the defense of functional residual capacity (13, $14,24,33)$. Our observations in control conditions are concordant with previous observations, including the lower percentage of breaths with TA EMG in REM sleep versus wakefulness and nREM sleep in both groups of lambs, and the higher percentage of breaths with TA EMG in older versus younger lambs $(14,24,34)$. This has been previously hypothesized to be related to the need to defend functional residual capacity against bulky intra-abdominal viscera at this age (24). Sleep deprivation did not have any significant effect on the percentage of breaths with TA expiratory EMG, except in REM sleep in younger lambs. We suggest that this small but significant increase in the percentage of breaths with TA expiratory EMG in REM sleep in younger lambs is further evidence of a compensatory mechanism for preserving end expiratory lung volume in the presence of an increased apnea index. This would be in keeping with the increase in sigh index observed in REM sleep in younger lambs after sleep deprivation, and could be triggered by the potent vagal feedback in the early postnatal period (24).

We have previously reported the presence of continuous TA EMG throughout spontaneous apneas in lambs, with complete glottal closure and maintenance of a high lung volume during apneas (i.e. inspiratory breath holding) $(14,35)$. We believe 
that this enhances gas exchange in the absence of alveolar ventilation (unpublished observations). In control conditions, the number of apneas with TA clearly tended to be higher (about 2-fold) in older than in younger lambs, regardless of the state of arousal. Again, this may be related to the need to further defend functional residual capacity against bulky intraabdominal viscera at this age (24). Sleep deprivation did not have any significant influence on the number of apneas with TA in younger lambs. Conversely, there was a clear tendency toward a decrease during wakefulness and nREM sleep in older lambs, which may have a deleterious effect on gas exchange during apneas. The absence of oxygenation recording within our radiotelemetry equipment at the time of the study unfortunately prevented us from testing this hypothesis.

\section{CONCLUSION}

In conclusion, our results in lambs suggest that the consequences of sleep deprivation upon respiration are predominant early after birth, at an age when respiratory instability is at its highest. Over and above their physiologic relevance, such observations are of clinical importance, for they represent a further incentive to refine our environmental interventions in early life, especially in neonatal care units. Finally, our data document that the freely moving, nonsedated neonatal lamb studied by telemetry is an appropriate model for sleep studies in the neonatal period.

Acknowledgments. The authors thank Bruno Gagné for expert technical assistance.

\section{REFERENCES}

1. Bowes G, Woolf GM, Sullivan CE, Phillipson EA 1980 Effect of sleep fragmentation on ventilatory and arousal responses of sleeping dogs to respiratory stimuli. Am Rev Respir Dis 122:899-908

2. White DP, Douglas NJ, Pickett CK, Zwillich CW, Weil JV 1983 Sleep deprivation and the control of ventilation. Am Rev Respir Dis 128:984-986

3. Leiter JC, Knuth SL, Bartlett Jr D 1985 The effect of sleep deprivation on activity of the genioglossus muscle. Am Rev Respir Dis 132:1242-1245

4. Chen HI, Tang YR 1989 Sleep loss impairs inspiratory muscle endurance. Am Rev Respir Dis 140:907-909

5. Spengler CM, Shea SA 2000 Sleep deprivation per se does not decrease the hypercapnic ventilatory response in humans. Am J Respir Crit Care Med 161:11241128

6. Schiffman PL, Trontell MC, Mazar MF, Edelman NH 1983 Sleep deprivation decreases ventilatory response to $\mathrm{CO}_{2}$ but not load compensation. Chest 84:695-698
7. Kahn A, Franco P, Scaillet S, Grosswater J, Dan B 1997 Development of cardiopulmonary integration and the role of arousability from sleep. Curr Opin Pulm Med $3: 440-444$

8. Canet E, Gaultier C, D'Allest AM, Dehan M 1989 Effects of sleep deprivation on respiratory events during sleep in healthy infants. J Appl Physiol 66:1158-1163

9. Thomas DA, Poole K, McArdle EK, Goodenough PC, Thompson J, Beardsmore CS, Simpson H 1996 The effect of sleep deprivation on sleep states, breathing events, peripheral chemoresponsiveness and arousal propensity in healthy 3 month old infants. Eur Respir J 9:932-938

10. Davis GM, Moscato J 1994 Changes in lung mechanics following sighs in premature newborns without lung disease. Pediatr Pulmonol 17:26-30

11. Thach BT, Taeusch Jr HW 1976 Sighing in newborn human infants: role of inflation-augmenting reflex. J Appl Physiol 41:502-507

12. Kianicka I, Praud J-P 1997 Influence of sleep states on laryngeal and abdominal muscle response to upper airway occlusion in lambs. Pediatr Res 41:862-871

13. Fisher JT, Mortola JP, Smith JB, Fox GS, Weeks S 1982 Respiration in newborns: development of the control of breathing. Am Rev Respir Dis 125:650-657

14. Renolleau S, Létourneau P, Niyonsenga T, Praud J-P 1999 Thyroarytenoid muscle electrical activity during spontaneous apneas in preterm lambs. Am J Respir Crit Care Med 159:1396-1404

15. Bureau MA, Bégin R 1982 Postnatal maturation of the respiratory response to $\mathrm{O}_{2}$ in awake newborn lambs. J Appl Physiol 52:428-433

16. Kianicka I, Leroux JF, Praud J-P 1994 Thyroarytenoid muscle activity during hypocapnic central apneas in awake nonsedated lambs. J Appl Physiol 76:1262-1268

17. Létourneau P, Dumont S, Kianicka I, Diaz V, Dorion D, Drolet R, Praud J-P 1999 Radiotelemetry system for apnea study in lambs. Respir Physiol 116:85-93

18. Zeger SL, Liang KY 1986 Longitudinal data analysis for discrete and continuous outcomes. Biometrics 42:121-130

19. Frank MG, Morrissette R, Heller HC 1998 Effects of sleep deprivation in neonatal rats. Am J Physiol 275:R148-157

20. Benington JH, Heller HC 1994 Does the function of REM sleep concern non-REM sleep or waking? Prog Neurobiol 44:433-449

21. Davey MG, Johns DP, Harding R 1998 Postnatal development of respiratory function in lambs studied serially between birth and 8 weeks. Respir Physiol 113:83-93

22. Arsenault J, Carrier E, Brien M, Praud J-P 2001 Postnatal development of vagal control of ventilation in preterm lambs. Am J Respir Crit Care Med 163:241A(abstr)

23. Martin RJ, Miller MJ, Carlo WA 1986 Pathogenesis of apnea in preterm infants. J Pediatr 109:733-741

24. Harding R, Johnson P, McClelland ME 1980 Respiratory function of the larynx in developing sheep and the influence of sleep state. Respir Physiol 40:165-179

25. Neilly JB, Kribbs NB, Maislin G, Pack AI 1992 Effects of selective sleep deprivation on ventilation during recovery sleep in normal humans. J Appl Physiol 72:100-109

26. Horne JA 1977 The effect of sleep deprivation upon variations in heart rate and respiration rate. Experientia 33:1175-1177

27. Kato M, Phillips BG, Sigurdsson G, Narkiewicz K, Pesek CA, Somers VK 2000 Effects of sleep deprivation on neural circulatory control. Hypertension 35:1173-1175

28. Nicholas TE, Power JH, Barr HA 1982 The pulmonary consequences of a deep breath. Respir Physiol 49:315-324

29. Issa FG, Porostocky S 1993 Effect of sleep on changes in breathing pattern accompanying sigh breaths. Respir Physiol 93:175-187

30. Jeffery HE, Megevand A, Page M 1999 Why the prone position is a risk factor for sudden infant death syndrome? Pediatrics 104:263-269

31. Lichter I, Muir RC 1975 The pattern of swallowing during sleep. Electroencephalogr Clin Neurophysiol 38:427-432

32. Sumi T 1963 The activity of brain-stem respiratory neurons and spinal respiratory motoneurons during swallowing. J Neurophysiol 26:466-477

33. England SJ, Kent G, Stogryn HA 1985 Laryngeal muscle and diaphragmatic activities in conscious dog pups. Respir Physiol 60:95-108

34. Harding R 1986 The upper respiratory tract in perinatal life. In: Johnston BM, Gluckman PD (eds) Reproductive and Perinatal Medicine (III), Respiratory Control and Lung Development in the Fetus and Newborn. Perinatology Press, Ithaca, NY, pp 331-376

35. Lemaire D, Létourneau P, Dorion D, Praud J-P 1999 Complete glottic closure during central apnea in lambs. J Otolaryngol 28:13-19 